Archives

$4 \mid 1989$

Varia

Techniques et organisation en chine : l'exemple de l'irrigation et de la protection hydraulique a l'époque impériale

\title{
Pierre-Étienne Will
}

\section{(2) OpenEdition \\ Journals}

Édition électronique

URL : http://journals.openedition.org/ccrh/2904

DOI : $10.4000 /$ ccrh. 2904

ISSN : $1760-7906$

Éditeur

Centre de recherches historiques - EHESS

Édition imprimée

Date de publication : 15 octobre 1989

ISSN : 0990-9141

Référence électronique

Pierre-Étienne Will, «Techniques et organisation en chine : l'exemple de l'irrigation et de la protection hydraulique a l'époque impériale », Les Cahiers du Centre de Recherches Historiques [En ligne], 4 | 1989, mis en ligne le 13 avril 2009, consulté le 19 avril 2019. URL : http://journals.openedition.org/ccrh/2904 ; DOI : 10.4000/ccrh.2904

Ce document a été généré automatiquement le 19 avril 2019

Article L.111-1 du Code de la propriété intellectuelle. 


\title{
Techniques et organisation en chine : l'exemple de l'irrigation et de la protection hydraulique a l'époque impériale
}

\author{
Pierre-Étienne Will
}

1 Il m'a semblé intéressant d'aborder le thème retenu pour cette réunion en traitant d'un domaine où la Chine a quelque chance de présenter une situation et des solutions originales. Les pages qui suivent sont donc consacrées aux problèmes d'irrigation et de protection hydraulique, et je les considérerai plus particulièrement sous l'angle de l'articulation entre techniques et organisation. J'entends ici « techniques » au sens étroit (matériel) du terme, par opposition à celui plus large où l'organisation est elle-même une technique : ainsi du concept fructueux de «techniques d'encadrement» dans les travaux de Pierre Gourou.

2 La question de l'articulation entre techniques et organisation peut être envisagée sous deux angles, opposés d'une certaine manière. On peut se demander en quoi les formes d'organisation observables dans le monde chinois traditionnel, dont quelques-unes au moins lui sont spécifiques, ont eu un impact sur la façon dont certains choix techniques étaient mis en oeuvre et poursuivis. C'est ce que j'essayerai de faire ici. Mais on peut aussi poser la question dans l'autre sens et se demander en quoi les nécessités techniques déterminées par un environnement donné influent sur les formes d'organisation. Dans le domaine de l'irrigation, et des aménagements hydrauliques en général, c'est renvoyer à des théories bien connues associant certains types de sites - disons, les deltas inondables - avec certaines formes d'organisation socio-politique - disons, les despotismes bureaucratiques. Le plus illustre des théoriciens ayant travaillé en ce sens est bien sûr K. A. Wittfogel.

3 Autant préciser tout de suite que ce que j'ai appris de la Chine en général, et ce que j'ai étudié de ses systèmes hydrauliques en particulier ${ }^{1}$, me rend plus que sceptique sur l'utilité et sur la véracité du modèle wittfogélien. Il ne me semble pas que ce qu'on trouve 
sinon de despotique, à tout le moins d'autocratique ou simplement de centralisateur, et bien sûr de bureaucratique, en Chine à différentes époques découle de ce genre de détermination géographique et technologique.

4 Il y a à cela une raison de simple chronologie : on ne peut en aucun cas dire que les grands ensembles hydrauliques qui existent effectivement là-bas, et dont certains sont fort anciens, ont façonné les constituants essentiels de l'État centralisé à la chinoise. Ils viennent après, et s'il faut vraiment établir une relation de cause à effet, celle-ci opérerait plutôt dans l'autre sens. Ce sont les exigences de la formation impériale en termes de productivité fiscale et de logistique qui l'ont conduite, dans certains cas et sur certains sites, à oeuvrer pour la création de ces dispositifs, lesquels ne lui sont nullement consubstantiels; et ce sont les possibilités qu'elle offrait en matière d'organisation qui ont, dans une certaine mesure, favorisé leur développement et ont aidé à leur maintien en ordre de marche. Mais dans une certaine mesure seulement, car on constate la plupart du temps l'existence de forces qui, bien qu'elles soient partie intégrante de la formation socio-politique dominante, oeuvrent en fait au détriment de ce maintien. Ce qui déjà devrait suffire à suggérer que la question est nettement plus complexe qu'on ne le pense parfois.

\section{Construction et entretien}

On trouvera une formulation particulièrement satisfaisante pour introduire le genre de problème dont il est ici question dans une étude récemment parue, consacrée non à la Chine mais à l'approvisionnement des villes dans le Proche-Orient ancien ${ }^{2}$. Cette étude comporte en effet un passage intéressant sur l'irrigation, où l'auteur insiste sur la différence fondamentale entre les fonctions de construction d'une part, et les fonctions de gestion et d'entretien (de «maintenance », pourrions-nous dire) de l'autre. D'après lui la recherche comparée montre que, si les premières requièrent généralement l'intervention d'une bureaucratie plus ou moins centralisée, les secondes en revanche sont mieux remplies par les producteurs eux-mêmes, même quand il s'agit de grands systèmes concernant des milliers d'unités de production. Qu'il s'agisse de répartir les ressources en eau ou d'entretenir les dispositifs, les gestionnaires autochtones ont l'avantage d'être familiers avec le terrain, la population, les méthodes agricoles, les types de conflits qui risquent de se produire, et pour cette raison ils se révèlent toujours plus efficaces que des bureaucrates sans compétence spécialisée et sans implantation locale. Autrement dit, l'existence d'une bureaucratie centralisée, avec son cérémonial et ses techniques de coercition, serait superflue à partir du moment où c'est l'exploitation d'un système existant qui est concernée.

6 Ailleurs, le même auteur introduit le concept intéressant d'entropie. L'entropie menace tout système d'irrigation: entendons par là une sorte de processus inévitable de fragilisation des digues, d'envasement des canaux, d'accumulation des conflits portant sur l'utilisation des ressources (de l'eau) et la répartition des charges d'entretien. C'est pour enrayer ce processus que des structures stables de maintenance apparaissent indispensables.

7 Dans cette acception, la tendance à l'entropie s'observe partout dans les exemples chinois que j'ai étudiés ; mais on verra aussi que l'idée suivant laquelle les meilleurs agents pour la combattre sont des agents non-bureaucratiques ne s'applique pas telle quelle. Sans avoir, et de loin, la présence et la domination « wittfogéliennes » qu'on pourrait être 
tenté de lui imaginer, la bureaucratie étatique n'en conserve pas moins un rôle clé, et c'est ce qui me conduira en fin de parcours à introduire, avec celle d'entropie, la notion de « cycle hydraulique/bureaucratique ».

\section{Trois types de bassins dans la vallée du Yangzi}

8 Les techniques de contrôle des eaux peuvent être regroupées en quelques catégories générales que l'on retrouve dans à peu près tous les environnements chinois (et, cela va sans dire, dans bien d'autres environnements): techniques d'irrigation (réservoirs, retenues, canaux, vannes, puits, norias...) ; techniques de protection (digues) ; techniques de drainage (systèmes de canaux et de trop-pleins). Pour en étudier l'articulation avec les problèmes d'organisation, je tirerai mes exemples de trois ensembles hydrauliques réunissant entre eux un vaste assortiment de combinaisons. Tous les trois se situent dans le drainage du Yangzi :

\section{Les polders du delta inférieur}

9 Ce qu'on appelle couramment le « delta du Yangzi » n'est pas à proprement parler le cône de déjection du fleuve: il s'agit en fait du drainage du lac Tai, lequel se déverse dans l'estuaire du Yangzi par un certain nombre de rivières (en l'occurrence les affluents de droite du Yangzi situés le plus en aval). La plaine traversée par ces dernières est absolument plate et située à quelques mètres d'altitude; les gradients d'écoulement très faibles favorisent un alluvionnement intense ; enfin, l'évacuation vers l'estuaire est gênée par un relèvement de la topographie et par la marée océanique. Dans sa version aménagée, en existence depuis le $10^{\mathrm{e}}$ siècle, le drainage de ce qu'on appelle aussi la plaine du Jiangnan (le pays au sud du Fleuve) s'effectue par l'intermédiaire d'un réseau très dense de canaux ranchés sur les rivières naturelles, elles-mêmes élargies et contenues entre des digues. Les rizières sont protégées derrières des petites digues fermées (yu) et, suivant les saisons, drainées ou irriguées par les canaux qui longent les digues: d'où l'appellation de « polders » donnée par la plupart des auteurs aux unités ainsi encloses.

Le problème technique majeur dans un tel site est l'évacuation continue des excès d'eau et la lutte contre l'alluvionnement; et le principal problème d'organisation est la répartition correcte des charges et des responsabilités, tant pour l'entretien annuel des polders et des canaux (au niveau des villages) que pour la remise en état périodique du système global de drainage et de protection (au niveau des sous-préfectures, des préfectures et du gouvernement provincial).

\section{L'aménagement de la plaine du Huguang}

11 Autrement dit de ce qui constitue une sorte de "delta intérieur» du Yangzi (correspondant au bassin central des provinces du Hubei et du Hunan). Là aussi on parle parfois de polders, mais la forme et la fonction des dispositifs sont un peu différentes de ce qu'on a dans le bas Yangzi. Autour du lac Dongting (au Hunan), les digues circulaires ou «enceintes» (wei) permettent de gagner de riches terrains alluviaux sur le lac et protègent les rizières en saison de hautes-eaux. Sur la rive gauche (au Hubei), beaucoup plus exposée aux crues brutales du fleuve et de son principal affluent de gauche, la Han, les enceintes (appelées yuan) sont abritées derrière des systèmes de longues digues 
formant une première ligne de protection et rendant par là même possible la mise en valeur des marais et des fonds de lacs qui servent normalement de réservoirs aux crues saisonnières. Les enceintes du Hunan et plus encore celles du Hubei sont en général de dimensions beaucoup plus vastes que les polders du Jiangnan.

Le problème technique majeur au nord du Yangzi est la protection des dispositifs contre des crues violentes et soudaines, ainsi que le stockage de ces crues avant évacuation, alors qu'autour du lac Dongting c'est la protection des cultures contre une montée des eaux en général beaucoup plus lente, et le drainage de ces hautes-eaux vers le Yangzi par le goulet situé en aval, à proximité de la cité de Yuezhou.

13 Sur le plan de l'organisation on retrouve le même type de dualité qu'au Jiangnan entre échelon local et échelon régional, avec cette différence que les unités de base (les " enceintes ») sont des ouvrages en général plus importants, englobant des communautés plus nombreuses, et pour certains d'entre eux placés sous le contrôle direct de la buraucratie locale.

\section{Les dispositifs d'irrigation en montagne dans le bassin supérieur de la Han}

Cet ensemble, qui correspond administrativement au Sud de la province du Shaanxi, est très différent des deux précédents, ne serait-ce que par l'aspect compartimenté imposé par la topographie. L'irrigation s'effectue occasionnellement au moyen de puits ou de réservoirs, mais le système dominant est la captation des cours d'eau: la configuration type comprend un barrage en amont, qui détourne l'eau vers des canaux courant à flanc de coteau et contenus derrière une digue aval (le canal comme la digue sont souvent désignés par le terme yan), avec une série de bouches de décharge servant à irriguer les fonds de vallée et les petits bassins où la riziculture est possible. La plupart de ces dispositifs sont d'échelle locale, mais certains possèdent un rayon d'action couvrant plusieurs sous-préfectures (typiquement, deux), ce qui soulève des problèmes particuliers de coordination.

15 Le principal problème technique se posant en montagne - outre le design de départ, parfois très complexe - est la fragilité de ces installations dans un environnement où le déboisement et les défrichements incontrôlés déstabilisent les sols des drainages supérieurs et où une pluie d'orage peut gonfler les torrents au point de tout emporter.

Les problèmes organisationnels sont en partie les mêmes que dans les bassins alluviaux décrits ci-dessus : celui en particulier de la répartition des charges d'entretien entre les différentes communautés bénéficiant d'un aménagement donné, ou entre les différents bénéficiaires à l'intérieur d'une même communauté. En revanche la question de la répartition de l'eau pour l'irrigation - concrètement, du contrôle des périodes d'ouverture des bouches de décharge - se pose ici de façon plus aiguë que dans les bassins inférieurs, où en général c'est plutôt l'excès d'eau à évacuer (le drainage) qui pose problème.

17 Si l'on reprend à présent l'opposition entre fonctions de construction et fonctions de " maintenance » évoquée plus haut, les deux premiers environnements décrits à l'instant se distinguent assez nettement du troisième. C'est ce que je vais essayer de détailler dans les paragraphes qui suivent. 


\section{La construction des sites}

18 a) Historiquement, l'aménagement du Jiangnan comme celui de la plaine du Huguang sont partis d'un plan initial à grande échelle, lancé par une autorité étatique centralisée. Dans le cas du Jiangnan, c'est au pouvoir régional du Wu-Yue, pendant la période de fragmentation politique des Cinq Dynasties et Dix Royaumes au 10e siècle, qu'est attribuée la mise en place du système des « polders et canaux » sur lequel repose la mise en valeur intensive du drainage du lac Tai - même si un resserrement considérable du réseau d'irrigation et de protection s'est opéré sous le régime des Song qui unifie l'empire à la fin du même siècle.

Dans le cas du moyen Yangzi (s'agissant en particulier de la plaine du Jiang-Han dans la moitié nord du Huguang), si l'on peut également parler d'un «plan» dont l'initiative revient aux instances supérieures de l'État, celui-ci s'est exécuté en plusieurs étapes distinctes, par ajout de certaines unités. Les premières longues digues sur la rive gauche du Yangzi, destinées à rendre exploitable la plaine inondable, et les premiers " caissons » servant à en régler le drainage dans sa partie occidentale, datent des Song du Sud (XII ${ }^{\mathrm{e}}$-XIII ${ }^{e}$ siècles); de nouveaux endigages ont été ajoutés ou reconstruits au début des Ming (fin XIV ${ }^{e}$ siècle - début xve), tandis qu'un autre élément essentiel de l'aménagement de la plaine, l'endigage continu de la Han, date du début du Xvi ${ }^{\mathrm{e}}$ siècle.

Cette notion de «plan » initial doit être précisée. Dans chacun des cas étudiés, l'action du gouvernement est partie d'un projet global de développement ayant pour objet une région inexploitée ou dévastée, et ce, même si tous les éléments futurs n'étaient pas anticipés dès le départ, ce qui est en particulier le cas dans le bassin du moyen Yangzi. Mieux, ce projet de développement, au stade initial du moins, incluait une visée stratégique. C'est clair dans le cas du moyen Yangzi, puisque l'objectif des Song méridionaux était d'y constituer une sorte de glacis face aux États barbares qui occupaient la Chine du Nord (les Jürchen, puis les Mongols) en peuplant et en développant la région de telle sorte qu'elle puisse ravitailler des forces armées suffisantes. Quoique moins explicite, l'objectif stratégique n'en existe pas moins dans le cas du Jiangnan sous le royaume de Wu-Yue: ce n'est pas une coïncidence si l'aménagement systématique du delta a été décidé pendant une période d'intense compétition entre des États régionaux cherchant à développer simultanément leurs forces productives, leur poids commercial et leurs capacités de résistance militaire (on compare parfois la Chine du $10^{\mathrm{e}}$ siècle à l'Europe classique).

D'un point de vue plus technique, il faut insister sur le fait que les régions considérées constituent des sites hydrographiquement intégrés où toute modification approfondie en un point du système retentit sur son économie générale: construction d'une digue modifiant le lit majeur d'un des principaux cours d'eau, fermeture d'un écoulement naturel, endigage et mise en culture d'une zone où s'épandent les inondations saisonnières... Ce qui rapproche également ces sites, c'est que ce sont originellement des plaines inondables soumises à un alluvionnement intense et dont l'hydrographie est très mouvante. Leur conversion en régions hautement productives passe nécessairement par un effort de stabilisation de cette hydrographie ; et cet effort, qui ne peut être réalisé au coup par coup, suppose des aménagements de grande ampleur dès le départ (les longues digues du moyen Yangzi et le réseau de drainage du Jiangnan). 
22 Pour revenir à la problématique esquissée au départ, on a, dans de tels cas, non pas un type d'environnement suscitant un type de formation étatique, mais un État préexistant, dont les formes et les ambitions s'enracinent dans une longue tradition, mettant en oeuvre ses capacités d'organisation pour faire d'une région jusqu'alors sous-développée une nouvelle base économique.

23 La domestication de ces sites vastes et hydrographiquement complexes pose d'immenses problèmes d'ingénierie, d'investissement et de mobilisation. Les trois aspects sont bien sûr inséparables. S'agissant d'ingénierie, il suffira d'évoquer les dimensions des canaux et des digues à réaliser, lesquelles peuvent atteindre des centaines de kilomètres dans les cas extrêmes; les cubages énormes de matériaux à remuer; toute la gamme des techniques de construction mises en oeuvre; plus encore, les problèmes de prévision et de coordination hydraulique au sein de réseaux très ramifiés, dont les débits et les pentes doivent être calculés sur de longues distances et avec des gradients très faibles, les vannes et les écluses à disposer, et le personnel spécialisé à mettre en place pour la gestion de structures dont les points stratégiques peuvent être très éloignés les uns des autres.

Les problèmes d'investissement financier et de mobilisation de la force de travail sont étroitement associés dans la mesure où l'administration cherche à limiter autant que possible la part des mises de fonds directes dans le coût global des travaux. Conformément au principe de mobilisation et d'affectation locale des ressources qui a presque toujours eu la préférence des régimes impériaux en Chine, l'idée est de faire contribuer et de mettre au travail en premier lieu les populations intéressées : ce sont donc des techniques essentiellement fiscales qui sont mises en oeuvre (au sens large, incluant les prestations en travail). On notera cependant une exception intéressante, encore que réservée en général à des travaux partiels de reconstruction : les opérations dites de "secours en échange de travail» (yi gong dai zhen) auxquelles procède parfois l'administration en année de crise de subsistances, souvent financées par des fonds extérieurs à la région concernée. Et l'on notera aussi, surtout à des époques plus anciennes (les Song ou le début des Ming) où l'État dispose de forces armées très nombreuses, la possibilité de recourir à la force de travail militaire.

Dans tous les cas, dès lors qu'il s'agit de mobiliser une force de travail importante, les techniques d'encadrement où excellent la bureaucratie chinoise et les réseaux d'administration rurale qui la prolongent prennent une place prépondérante et permettent à la machine de tourner efficacement.

b) Par contraste avec les bassins décrits à l'instant, les systèmes d'irrigation du drainage supérieur de la Han sont dispersés, indépendants les uns des autres, et remontent à des dates très diverses (parfois fort anciennes). Ils découlent dans la majorité des cas d'initiatives bureaucratiques locales. Du moins est-ce ainsi que les principales sources dont nous disposions (les « monographies locales » compilées à l'échelon des préfectures et des sous-préfectures) présentent les choses : typique est la notice consacrée à un dispositif donné où l'on voit un magistrat activiste " constater la misère du peuple » dans sa nouvelle circonscription, inspecter le terrain, dresser les plans pour le canal ou la retenue qui permettront à une vallée de décoller économiquement, mobiliser la force de travail disponible, et enfin fixer les règles d'utilisation et d'entretien de la nouvelle installation, le tout étant dûment consigné dans un texte gravé sur une stèle.

Comme celle des grands ensembles hydrauliques, donc, la réalisation de ces aménagements locaux passe par un effort qui est en priorité de planning technique et de 
mobilisation. La consultation directe des intéressés joue un rôle d'autant plus important que, d'une part, ce sont eux qui versent les fonds nécessaires (en général sous forme de surtaxes s'ajoutant à l'impôt foncier, mais aussi par le biais des contributions exceptionnelles demandées aux riches) et qui fournissent le travail ; et que, d'autre part, l'on ne peut pas faire jouer, comme dans les grands sites intégrés, l'argument d'autorité en invoquant l'intérêt (c'est-à-dire la sécurité) d'une région entière : les tractations se déroulent à beaucoup plus petite échelle.

Suivant que l'on a affaire à de vastes bassins alluviaux ou à des vallées montagnardes, les aspects politiques et organisationnels sont donc différents. Mais il existe un facteur commun, qui possède ses traits et son intégration propres: c'est la bureaucratie impériale.

J'ai insisté sur la faiblesse usuelle des apports financiers publics extérieurs, c'est-à-dire provenant directement du budget de l'État. Pourtant ces aides, même partielles et remboursables, apparaissent souvent décisives pour rendre possible le démarrage des projets: on en trouve de nombreux exemples dans l'histoire du développement du haut bassin de la Han, et aussi bien dans les bassins inférieurs lorsqu'il s'agit d'additions locales à des ensembles déjà constitués. Probablement faut-il faire une différence entre le " temps de paix ", où les ponctions massives et autoritaires de travail corvéable ne sont guère envisageables - où tout se paie, si l'on veut -, et les situations de construction dynastique ou de défense frontalière auxquelles correspond la création des grandes infrastructures décrites plus haut.

L'aspect souligné à l'instant - le caractère décisif des aides financières publiques, même limitées - s'explique par un contexte de très basse capitalisation rurale et d'agriculture à faibles surplus; elle s'explique aussi par le fait que les décisions d'infrastructure (ou de reconstruction) sont souvent prises dans des situations de crise conjoncturelle, ou suivant la formule consacrée, de « population affaiblie».

\section{La gestion courante et la maintenance}

31 Si l'on se tourne à présent vers les problèmes de gestion courante et d'entretien des installations, on constate une semblable opposition entre les deux premiers environnements décrits et le troisième; mais on trouve à nouveau certains points communs.

Il me semble qu'une des distinctions essentielles provient de ce que, pour des raisons à la fois topographiques et hydrographiques, les grands systèmes deltaïques sont expansifs, alors que les petits systèmes montagnards sont clos sur eux-mêmes, définis dès le départ en quelque sorte. Le caractère « expansif» des grands systèmes est autant une question de densité que d'étendue spatiale. L'infrastructure de base créée par le pouvoir centralisé (canaux et digues) constitue en quelque sorte un cadre d'irrigation, de protection et de drainage à l'échelle globale du bassin, qui en rend ensuite possible le remplissage graduel et la mise en culture de plus en plus serrée.

Dans le cas du bas comme du moyen Yangzi, l'unité «technique» de mise en valeur agricole est la digue fermée (ou enceinte), disons le " polder ", bien que, comme on l'a vu, le terme implique une fonction permanente de drainage et convienne surtout dans le cas du bas Yangzi. Le développement est impulsé au départ par la construction de polders sous l'égide de l'administration; leur entretien est régi par des règles contraignantes, et 
financé par les contributions annuelles de propriétaires dûment recensés. Or l'espace ainsi aménagé laisse vacantes de vastes zones fonctionnant comme trop-pleins pour les crues saisonnières. L'histoire de ces bassins - je n'entre pas ici dans le détail de la chronologie - est celle de l'occupation progressive des espaces libres par de nouvelles exploitations, protégées ou non par des digues fermées, et créées par des entrepreneurs purement privés. On pourrait parler d'une phase de construction secondaire.

Le problème est que, d'une part, ces développements mettent progressivement en danger la sécurité de l'ensemble du système en compromettant les fonctions de drainage (en surchargeant le site, si l'on veut); et que d'autre part ils échappent plus ou moins au contrôle de l'administration, si bien que leurs auteurs réussissent largement à se soustraire à l'effort collectif d'entretien annuel (et, plus généralement, à l'impôt) : à terme l'on assiste à une excroissance démesurée, à l'échelon de communautés entières, $\mathrm{du}$ phénomène dit du free rider.

La conséquence est partout mentionnée dans les sources : les free riders en question sont ceux qui s'enrichissent, alors que les occupants des anciennes installations (autorisées), sur qui retombe tout le poids de la maintenance, s'appauvrissent et remplissent par conséquent de moins en moins bien leurs tâches d'entretien. C'est là un aspect fondamental, dans ce type de site, du phénomène d'entropie mentionné plus haut. Il n'est qu'en apparence paradoxal que cette «entropie » passe par le développement de forces centrifuges. Revenons-y plus en détail.

Deux facteurs en fait opèrent simultanément, et retentissent l'un sur l'autre : d'une part, la dégradation physique de l'environnement aménagé, et d'autre part la décadence des institutions et des procédures de gestion et d'entretien.

37 a) La dégradation "physique »: on pourrait aussi parler du caractère de plus en plus dangereux du cadre aménagé, qu'illustrent l'affaiblissement des digues, l'envasement des canaux, la dégradation des fonctions de drainage, la multiplication et l'aggravation des inondations... Ces évolutions soulignent évidemment la fragilité de tels systèmes et tiennent en partie, mais en partie seulement, à des facteurs qu'aucune organisation bureaucratique ne peut influencer : l'alluvionnement séculaire, les variations climatiques, la déforestation (contre laquelle les moyens d'action apparaissent historiquement dérisoires). Elles tiennent aussi à des facteurs qui ne sont guère influençables par voie administrative mais qui n'en sont pas moins d'origine " humaine ", et en outre locale et aisément discernable: la densification des cultures, la multiplication des endigages " privés » au détriment des équilibres généraux, dont les conséquences se manifestent de façon dramatique lorsque les crues saisonnières atteignent une ampleur exceptionnelle.

b) L'autre type de facteur, apparenté à ceux qui viennent d'être mentionnés, est non plus physique mais purement organisationnel : ce sont les dysfonctionnements des procédures de "gestion par la population sous la supervision de l'administration» mises en place après la phase de construction initiale.

39 Cette formule (guan $\mathrm{du}$ min ban) décrit exactement la philosophie du système de gouvernement à la chinoise : il s'agit d'une forme de délégation plus ou moins lâche, et l'on retrouve ici l'idée mentionnée au début de l'exposé suivant laquelle l'entretien des dispositifs hydrauliques, même de vastes dimensions, risque d'être mieux assuré lorsqu'il est confié aux occupants du site. Il faut cependant la nuancer. Dans les exemples chinois, la justification donnée par les sources est moins la familiarité des utilisateurs directs avec l'environnement physique et social que la nécessité de ne pas surcharger une 
bureaucratie régulière faible en effectifs et en moyens. Elle est aussi, très explicitement, qu'il faut limiter au maximum les exactions (considérées comme inévitables) des agents subalternes de l'administration. Cette dernière considération nous renvoie à l'un des problèmes structurels de l'administration territoriale chinoise depuis la dynastie des Song, qui est la dichotomie entre les fonctionnaires en titre (guan) et les agents d'exécution recrutés localement (désignés par des termes tels que $l i, x u, y i$, etc.), ces derniers étant beaucoup plus nombreux et très difficiles à contrôler.

On peut encore ajouter que le degré de supervision bureaucratique considéré comme désirable en Chine reste fonction de la taille des unités considérées et des problèmes techniques soulevés par leur gestion. Alors qu'au Jiangnan l'entretien et le fonctionnement $d u$ " polder » sont entièrement laissés à la communauté des utilisateurs, les longues digues qui bordent le Yangzi au Hubei sont surveillées de beaucoup plus près par les fonctionnaires des unités sous-préfectorales; ces derniers assurent même une présence permanente à proximité de certains dispositifs dont l'état et le bon fonctionnement sont considérés comme vitaux pour la sécurité de la région. Et je ne parle pas des tournées d'inspection régulières que les membres de la hiérarchie provinciale sont requis d'effectuer, au $18^{\mathrm{e}}$ siècle notamment.

41 Quoi qu'il en soit, même dans les cas où les remarques précitées de R. Hunt sur la supériorité des cadres locaux en matière d'entretien et de gestion apparaissent applicables, celle-ci n'est effective que dans le relativement court terme. En effet les structures d'« entretien par la population » qu'on retrouve un peu partout se révèlent périodiquement débordées par la situation et incapables d'assurer de façon autonome la simple conservation des systèmes dont elles ont la charge. C'est en particulier vrai à l'intérieur des grands sites.

Les raisons sont nombreuses. J'ai déjà fait allusion au simple alourdissement du risque physique: à partir d'un certain point, assez vite atteint, les communautés locales n'arrivent plus à adapter les techniques existantes d'irrigation et de protection hydraulique aux changements de l'environnement. Mais il y a aussi, en fait surtout, des raisons plus spécifiquement sociales ou même politiques. Partout le phénomène du free rider mentionné plus haut mine l'efficacité des structures locales de gestion hydraulique. On a vu qu'il ne fait qu'accélérer les processus de différenciation socio-économique, tout en concentrant les charges d'entretien sur les plus défavorisés. Dans le bas Yangzi s'y est ajoutée, pendant la deuxième moitié de la dynastie des Ming ( $\mathrm{XVI}^{\mathrm{e}}-\mathrm{XVII}{ }^{\mathrm{e}}$ siècles), l'émigration des propriétaires les plus riches vers les villes et leur désengagement progressif des tâches de maintenance annuelle au niveau des polders et des canaux, non seulement en termes de leadership mais aussi, et surtout, en termes de financement. En de nombreux endroits l'entretien des polders s'est retrouvé entre les mains de communautés de fermiers pauvres incapables de le prendre en charge, et de plus peu enclins à accepter le manque à gagner que représentait pour eux l'abandon sans contrepartie de leurs activités annexes d'artisanat pour remonter des digues ou draguer des canaux. La situation n'a pu être redressée qu'au début des Qing, au prix d'une réforme approfondie de la fiscalité rurale qui contraignait les propriétaires absentéistes à remplir leurs obligations. 


\section{Le cycle hydraulique/bureaucratique} par la superstructure bureaucratique sur les conditions locales de maintenance hydraulique : l'État n'intervient en force qu'à partir d'un certain degré de dégradation des procédures et des institutions existantes. Encore faut-il que les conditions politiques s'y prêtent. Dans le cas du Jiangnan il s'est écoulé plus d'un siècle (avant 1570-après 1670) entre le dernier effort d'intervention systématique dans l'hydraulique de la région sous la dynastie des Ming, et le premier sous celle des Qing: pendant ses dernières décennies d'existence l'appareil d'État des Ming était incapable de faire réellement face à la décadence des institutions d'entretien et au délabrement de l'infrastructure hydraulique qui en résultait, et ce n'est qu'après une certaine période de consolidation que la nouvelle dynastie a pu envisager de s'y attaquer. Les réformes fiscales auxquelles j'ai fait allusion avaient en fait été préparées par un certain nombre d'expériences dues à des fonctionnaires locaux activistes; mais l'imbrication des problèmes à l'intérieur du site rendait utopique toute solution autre que globale.

dans une situation normale, c'est-à-dire bien contrôlée par la bureaucratie, les désaccords s'expriment essentiellement par le canal de pétitions ou d'interventions auprès des autorités locales, à charge pour ces dernières de négocier avec les échelons supérieurs de l'administration provinciale, voire avec le pouvoir central. Dans de tels cas le découpage territorial apparait fréquemment comme un obstacle à la bonne gestion technique d'environnements dont les limites naturelles ne coïncident pas nécessairement avec celles des unités administratives, ou englobent plusieurs circonscriptions : outre que la communication horizontale tend plutôt à être découragée dans l'administration territoriale chinoise, les autorités locales sont naturellement portées à adopter et à défendre les intérêts exprimés par leurs populations, que ce soit pour des raisons de rendement fiscal ou pour des raisons d'opportunité politique, c'est-à-dire de bon voisinage avec les élites dont la collaboration leur est nécessaire pour gouverner. L'existence d'un pouvoir provincial et central fort, capable de résister aux pressions et d'imposer ses arbitrages, est donc une condition nécessaire pour que les dispositifs hydrauliques excédant un cadre purement local puissent fonctionner correctement.

Des considérations qui précèdent découle la notion de ce que j'ai appelé ailleurs « cycle hydraulique", mais qu'il serait plus correct de qualifier de cycle hydraulique/ bureaucratique. Partout la construction, le développement et la décadence des dispositifs d'irrigation, de protection et de drainage résultent du jeu combiné de l'entropie précédemment décrite et des efforts de la bureaucratie pour en combattre les effets. Dans la configuration la plus typique, la dégradation du fonctionnement hydraulique d'un site - par l'effet de la routine, des conflits, de la surcharge de l'environnement et de l'évolution naturelle de l'hydrographie - conduit à une situation de crise dont seule une intervention administrative exceptionnelle, directe et le plus souvent de haut niveau permet de sortir. Cette intervention met en oeuvre les techniques d'ingénierie, d'organisation, et les efforts d'investissement dont il a été question plus haut. La restructuration et la remise en ordre qui en résultent inaugurent un nouveau cycle.

46

Ce modèle, qui admet à coup sûr de nombreuses irrégularités, est bien en évidence dans le cas de l'aménagement du «delta » inférieur du Yangzi. On observe sous la dynastie des Ming, particulièrement, une succession de cycles couvrant approximativement un demi 
siècle, chaque fois conclus (ou inaugurés) par la remise en état du système d'évacuation des crues - concrètement, par le dragage, ou même la réouverture quand c'est nécessaire, des rivières qui drainent la région vers l'estuaire du Yangzi. Cette remise en ordre est décidée par le pouvoir central et supervisée par un haut commissaire dépêché par la Cour avec des pouvoirs et des moyens exceptionnels.

On peut parler d'un cycle de dimensions plus vastes lorsque, comme on l'a vu plus haut à propos de la même région, le pouvoir central n'a plus les moyens de prendre de telles mesures et laisse la situation se dégrader au delà des seuils habituels. Il arrive aussi que d'autres facteurs de désorganisation interviennent, qui débordent le cadre strict de la gestion hydraulique et sont parfois d'origine extérieure aux régions concernées. Le cycle hydraulique/bureaucratique peut alors coïncider avec ce qu'on est convenu d'appeler le cycle dynastique, dont il devient un des aspects les plus apparents.

Le phénomène est parfaitement en évidence dans le bassin moyen du Yangzi, à la fin de la dynastie des Ming en particulier, autrement dit au coeur de la crise générale du XvII ${ }^{\mathrm{e}}$ siècle. Au phénomène classique d'entropie, accentué dans ce cas par l'élargissement des défrichements et des endigages dangereux pour l'équilibre général du système, s'ajoutent l'incapacité du gouvernement des Ming à intervenir de façon drastique, l'accumulation séculaire des désastres naturels, et enfin, à partir des années 1630, le choc de la guerre civile et de l'invasion étrangère. Les armées rebelles qui parcourent en tous sens le Hubei et le nord du Hunan causent des dégâts considérables, tout comme d'ailleurs les armées officielles censées les combattre. L'impact est surtout sensible au niveau des structures d'entretien des endigages, complètement désorganisées par l'appauvrissement, quand ce n'est la fuite, des populations riveraines, et par la carence d'administrations locales absorbées par la guerre ou purement et simplement détruites par la rébellion. Laissés à l'abandon, digues, canaux et écluses tombent en ruine et de vastes superficies retournent à l'état de friches inondables.

Le pays dont prennent possession les Qing (officiellement dès 1645, mais avec des retours en arrière et de nombreux conflits jusque vers 1670) est fortement dépeuplé, l'aménagement du cadre naturel en grande partie détruit. Une fois son pouvoir affermi, l'action de la nouvelle administration va procéder de cette fonction de construction - en l'occurrence, de reconstruction - que j'ai opposée au début de cette étude à celle d'entretien, et enclencher par là un nouveau cycle de développement. La remise en place progressive des longues digues et des principales «enceintes », organisée et supervisée par la hiérarchie provinciale, consolidée par la redéfinition des procédures d'entretien, accompagne et encourage à la fois le repeuplement du bassin ; celui-ci est facilité par une immigration active en provenance des provinces situées plus en aval. Le même processus que pendant les siècles Ming s'instaure dès lors: surcharge progressive du site, construction d'installations non contrôlées par l'administration et dangereuses pour celles qui existent déjà, conflits de toute nature, relâchement des institutions chargées de la maintenance locale, sans parler du déboisement des drainages supérieurs à partir du milieu du $18^{\mathrm{e}}$ siècle et de ses effets en termes d'alluvionnement et de sautes de débit au moment des crues saisonnières.

La différence avec le cycle précédent est que la pression sur l'environnement est plus accentuée en raison de beaucoup plus fortes densités démographiques, mais aussi que la résistance de la superstructure bureaucratique au processus d'entropie apparaît plus efficace : sans entrer dans les détails, on dira simplement que la surveillance du terrain à tous les échelons est plus étroite, la coordination au sein de la hiérarchie mieux assurée, 
et les moyens d'intervention ponctuelle (financiers notamment) beaucoup plus importants. Même si la dégradation globale des équilibres du bassin conduit à de véritables désastres hydrauliques en 1788 et à nouveau en 1831, le pouvoir reste capable jusqu'à ces années d'en analyser les causes, de formuler et de mettre au moins partiellement en oeuvre des politiques cohérentes de remise en ordre. Un peu comme au moment de la transition Ming-Qing, c'est une série de bouleversements socio-militaires - l'invasion des Taiping dans les années 1850 - qui marque le point le plus bas du cycle et met un terme pour plusieurs décennies aux ambitions officielles de contrôle global du site et de ses aménageurs.

51 Autant que je puisse en juger (ma lecture des sources reste pour le moment partielle), la situation est un peu différente dans les périphéries montagneuses de la haute vallée de la Han. Les systèmes locaux y sont beaucoup plus indépendants les uns des autres que dans le cas des plaines alluviales, mieux définis dès le départ et, comme on l'a vu, moins expansifs. Les conflits y sont plus faciles à gérer, parce que circonscrits à l'intérieur d'aires géographiques limitées et dépendant rarement de plusieurs instances administratives. La pression démographique les affecte de façon indirecte dans la mesure où les défrichements qui les menacent leur sont extérieurs : ils opèrent en amont des sytèmes, sur les versants non irrigables dont ils détruisent durablement la couverture végétale. En fait le principal facteur cyclique semble être ici la guerre (ou la rébellion) et les dévastations qu'elle provoque ; mais chaque petit bassin a son histoire propre dans la mesure où les commotions politiques qui ponctuent celle des grands systèmes atteignent l'un mais peuvent épargner l'autre.

\section{Conclusion}

La configuration décrite dans ces pages fait intervenir trois niveaux : celui des techniques proprement dites, soumises dans le cas des grands systèmes à des contraintes particulières d'échelle et de coordination; celui de la maintenance routinière, laissée dans la majorité des cas à des organisations procédant directement des populations riveraines; et celui de la gestion bureaucratique, laquelle n'opère directement (et globalement) que de façon épisodique.

Cette configuration, où le rôle tenu par la superstructure bureaucratique me semble en parfaite conformité avec la nature même du système étatique chinois à la fin de l'empire, n'est cependant pas universelle. Il existe des grands ensembles hydrauliques placés directement et en permanence sous le contrôle d'une bureaucratie spécifique - à vrai dire il en existe essentiellement un : c'est le système constitué par le Grand Canal et le Fleuve Jaune. La grande différence avec les ensembles situés le long du Yangzi est qu'il ne s'agit pas ici d'irrigation et de défrichements, ou de façon secondaire seulement, mais du maintien d'une route de transport stratégique-la plus stratégique qui puisse être puisque c'est l'approvisionnement de la capitale et des armées de la frontière Nord qui est en cause. La fonction de protection est elle aussi, d'une certain façon, secondaire: l'endigage et la stabilisation du Fleuve Jaune sont d'abord indispensables à la survie du Canal.

54 L'étroite surveillance dont ce système est l'objet, le niveau très élevé de ses responsables (ils ont le même rang que les gouverneurs généraux des provinces) s'expliquent autant par son importance stratégique que par la difficulté extrême à le maintenir en état de fonctionnement. Le Canal est un tour de force technique et son interconnection avec une 
voie d'eau aussi capricieuse que le Fleuve Jaune semble en permanence un défi aux lois de l'équilibre. Mais l'analyse des problèmes très spéciaux d'ingénierie et d'organisation soulevés nous entraînerait trop loin : aussi bien s'agit-il d'un environnement entièrement différent de ceux qui ont été décrits dans ces pages.

\section{NOTES}

1. Cf. P.-E. WILL, «Un cycle hydraulique en Chine : la province du Hubei du XVI ${ }^{\mathrm{e}}$ au XIX ${ }^{\mathrm{e}}$ siècle ", Bulletin de l'École Française d'Extrême-Orient. LXVIII (1980), p. 261-287 : «Crise politique, crise des encadrements, crise hydraulique et crise démographique : la basse conjoncture dans le bassin central du Yangzi au XVII siècle", in Pierre GOUROU et Gilbert ETIENNE (éds.), Des labours de Cluny à la révolution verte: techniques agricoles et population, Paris, PUF, 1985, p. 63-84; "State intervention in the administration of a hydraulic infrastructure : the example of Hubei province in late imperial times ", in Stuart Schram (ed.), The Scope of State Power in China, Londres/Hong Kong: SOAS/Chinese University, 1985, p. 295-347; «The occurrence of, and response to, catastrophes and economic change in the Lower and Middle Yangzi, 1500-1850", in R. DERNBERGER, A. FEUERWERKER et R. HARTWELL (eds.), State and Enterprise in Chinese Economic History, 980-1980. Berkeley, University of California Press, à paraître ; « On state management of water conservancy in late imperial China », Papers on Far Eastern History [Canberra], 36 (1987), p. 71-91. On trouvera dans ces études les références aux sources et aux travaux sur lesquels s'appuient les considérations qui suivent. Robert C. HUNT, "The role of bureaucracy in the provisioning of cities : a framework for analysis of the ancient Near East ", in GIBSON et BIGGS (eds.), Bureaucracy in the Ancient Near East, Oriental Institute, University of Chicago, 1988, p. 161-92.

2. Robert C. HUNT, «The role of bureaucracy in the provisioning of cities : a framework for analysis of the ancient Near East », in GIBSON et BIGGS (eds.), Bureaucracy in the Ancient Near East, Oriental Institute, University of Chicago, 1988, p. 161-92.

\section{AUTEUR}

\section{PIERRE-ÉTIENNE WILL}

Pierre-Etienne WILL est directeur d'études au Centre de Recherche et de Documentation sur la Chine Contemporaine. 